\title{
Opioid-Related Harms in Canada
}

Vera Grywacheski, Shannon O'Connor and Krista Louie

\begin{abstract}
The rise in harms associated with opioids is an issue of increasing public health importance in Canada. The Government of Canada recently reported 2,816 apparent opioid-related deaths across the country in 2016. Recent 2017 data show that deaths involving fentanyl-related opioids have doubled from January to March as compared to the same time period in 2016 (Government of Canada 2017). Additional measures that provide a better understanding of opioid-related harms, such as hospitalizations and emergency department (ED) visits, are a high priority. The objective of this study is to present pan-Canadian data on hospitalizations and ED visits because of opioid poisoning.
\end{abstract}

Opioid poisoning refers to a potentially lethal harm that occurs when a person takes an opioid incorrectly. Opioid poisonings may occur as a result of a drug overdose, accidental ingestion or purposely self-inflicted harm including suicide attempts. Persons who are merely intoxicated with opioids or experiencing adverse effects from opioids that are correctly administered, such as an allergic reaction, are not included (CIHI 2015).

\section{Approach}

We used ICD-10-CA (v 2015) codes to identify opioid poisonings in hospital and emergency department (ED) data housed at the Canadian Institute for Health Information.

The hospitalization analysis included data from 2007-2008 to 2016-2017 from all provinces and territories (except Quebec and Nunavut, where the most recent data available at the time of the study were from 2015-2016). The analysis of hospital data was limited to cases in which opioid poisoning was considered influential to the time spent in hospital and treatment received by the patient while there.

The ED analysis was based on available, comparable data. At the time of the study, this included data from 2012-2013 to 2016-2017 from Ontario and Alberta. Although ED data were available for Yukon, it was excluded because of small volumes. The analysis of ED data included any documented diagnosis of opioid poisoning.

Population data from Statistics Canada were used to calculate rates.

\section{Key Findings \\ Hospitalizations because of opioid poisoning}

Opioid poisonings result in an average of 16 hospitalizations each day in Canada (Figure 1). Over the past 10 years, the rate of hospitalizations for opioid poisonings has increased by more than 50\%. Particularly concerning, nearly half the increase in hospitalizations occurred over the last three years.

\section{FIGURE 1.}

Average number of opioid poisoning hospitalizations per day in Canada, 2016-2017
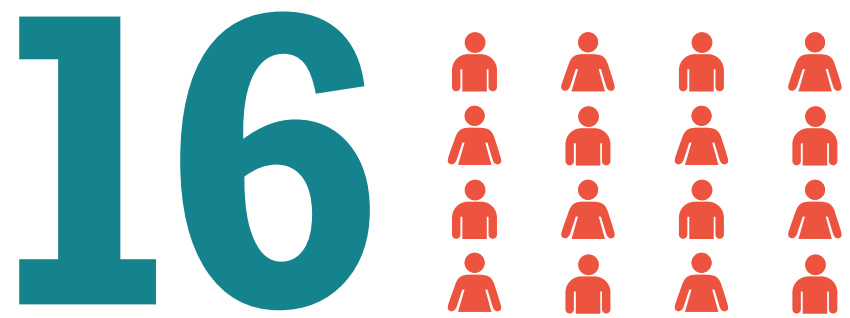

Note: Quebec and Nunavut data are from 2015-2016 (the most recent year of data available) Source: Hospital Morbidity Database, Canadian Institute for Health Information.

Examining the reason behind these opioid poisonings, we found that more than half were accidental while almost one-third were a result of purposely self-inflicted harm in 2016-2017.

With respect to age, we found that older adults aged 45-64 and seniors aged 65+ were particularly vulnerable, as they had the highest rates of hospitalizations. However, increases were seen across all age groups over the study period, with youth aged 15-24 and adults aged 25-44 having the fastest-growing rates.

Rates of hospitalization because of opioid poisoning varied across Canada, but are increasing in virtually all jurisdictions. In 2016-2017, rates were highest in British Columbia and Alberta (Figure 2).

Among census metropolitan areas (cities with a population of 100,000 or more), Kelowna, BC, and Brantford, ON, had the highest rates of hospitalization in 2016-2017. Other selected census metropolitan areas are presented in Figure 3. 
FIGURE 2.

Opioid poisoning hospitalization rates by province/territory, 2016-2017

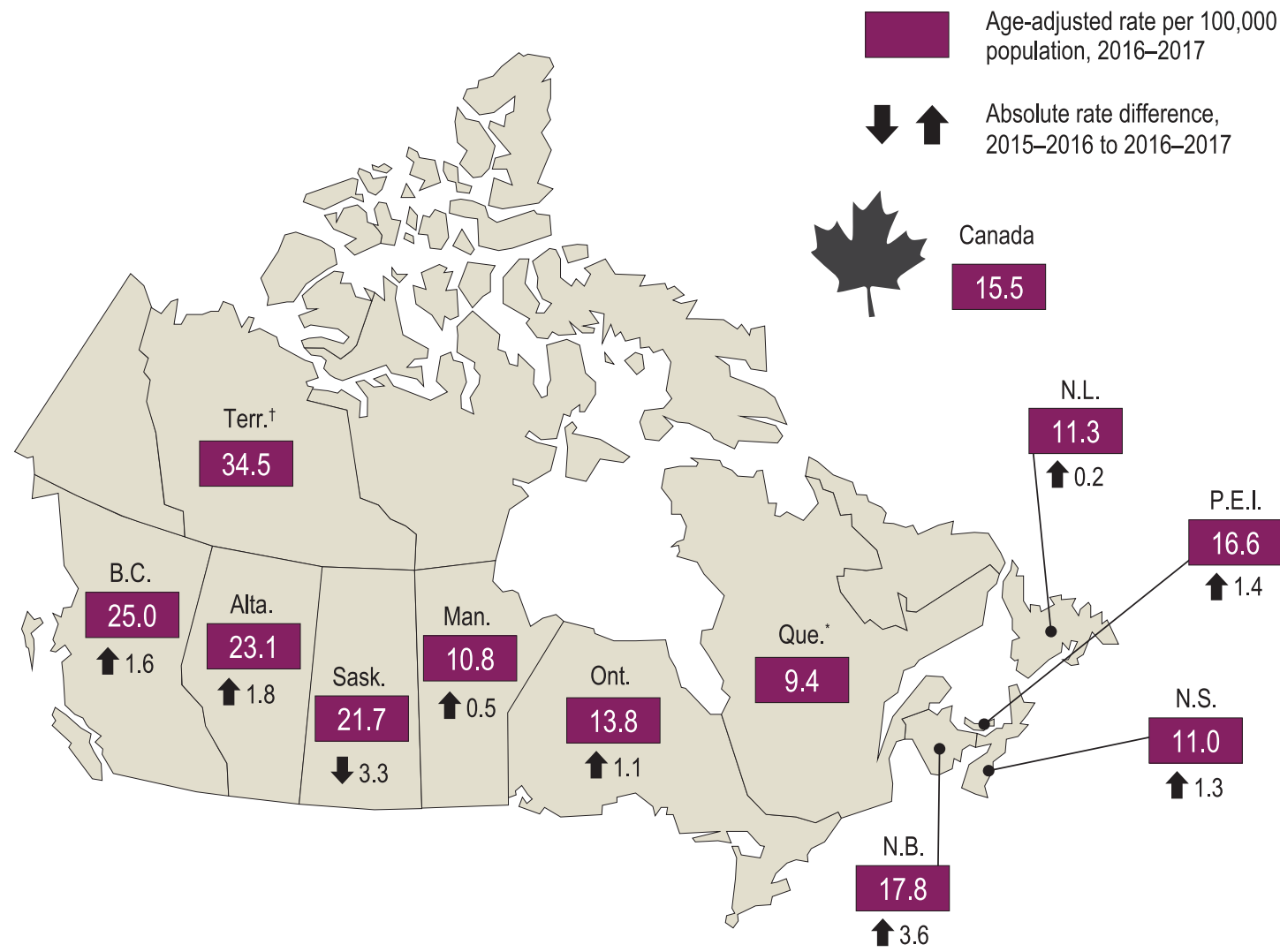

*Quebec data are from 2015-2016 (the most recent year of data available); therefore, there is no absolute rate difference shown.

†Yukon, Northwest Territories and Nunavut data are grouped because of low volumes. These data should be interpreted with caution. Nunavut data are from $2015-2016$ (the most recent year of data available); therefore, there is no absolute rate difference shown.

Source: Hospital Morbidity Database, Canadian Institute for Health Information.

\section{ED visits because of opioid poisoning}

Opioid poisonings result in an average of $13 \mathrm{ED}$ visits in Ontario and 11 ED visits in Alberta every day. Between 2012-2013 and 2016-2017, rates of ED visits more than doubled in Alberta and increased by almost $50 \%$ in Ontario. As with hospitalizations, the majority of the increase in $\mathrm{ED}$ visits occurred over the past three years.

We found that dramatic increases in heroin and synthetic opioid poisonings (including those related to fentanyl) were largely accountable for the rise in ED visits. In Alberta, the number of $\mathrm{ED}$ visits increased almost 10 -fold for heroin poisonings and more than 10-fold for synthetic opioid poisonings over the past 5 years. In Ontario, ED visits increased almost fourfold for heroin poisonings and more than doubled for synthetic opioid poisonings.

In Alberta, youth aged 15-24 had the highest and fastest-growing rates of ED visits, tripling over the past 5 years. In Ontario, adults aged 25-44 had the highest and fastest-growing rates of ED visits, increasing by $85 \%$ during the study period.

\section{Additional findings}

Detailed findings for opioid poisoning hospitalizations and ED visits - as well as more information on data sources, terminology and methods - are described in a recent CIHI publication Opioid-Related Harms in Canada (2017). The publication and accompanying data tables are available at: https://secure.cihi.ca/ estore/productFamily.htm?pf $=\mathrm{PFC} 3520 \&$ lang $=$ en $\&$ media $=0$.

\section{Moving Forward}

These findings highlight the increasing harms from opioid poisonings across Canada. In moving forward, it is important to continue monitoring trends and to evaluate the impact of interventions targeted at reducing opioid-related harms. CIHI intends to publish updated analyses as more data become available, including $\mathrm{ED}$ data for more jurisdictions. $\mathrm{HQ}$ 


\section{FIGURE 3.}

Opioid poisoning hospitalization rates by selected census metropolitan areas, *2016-2017

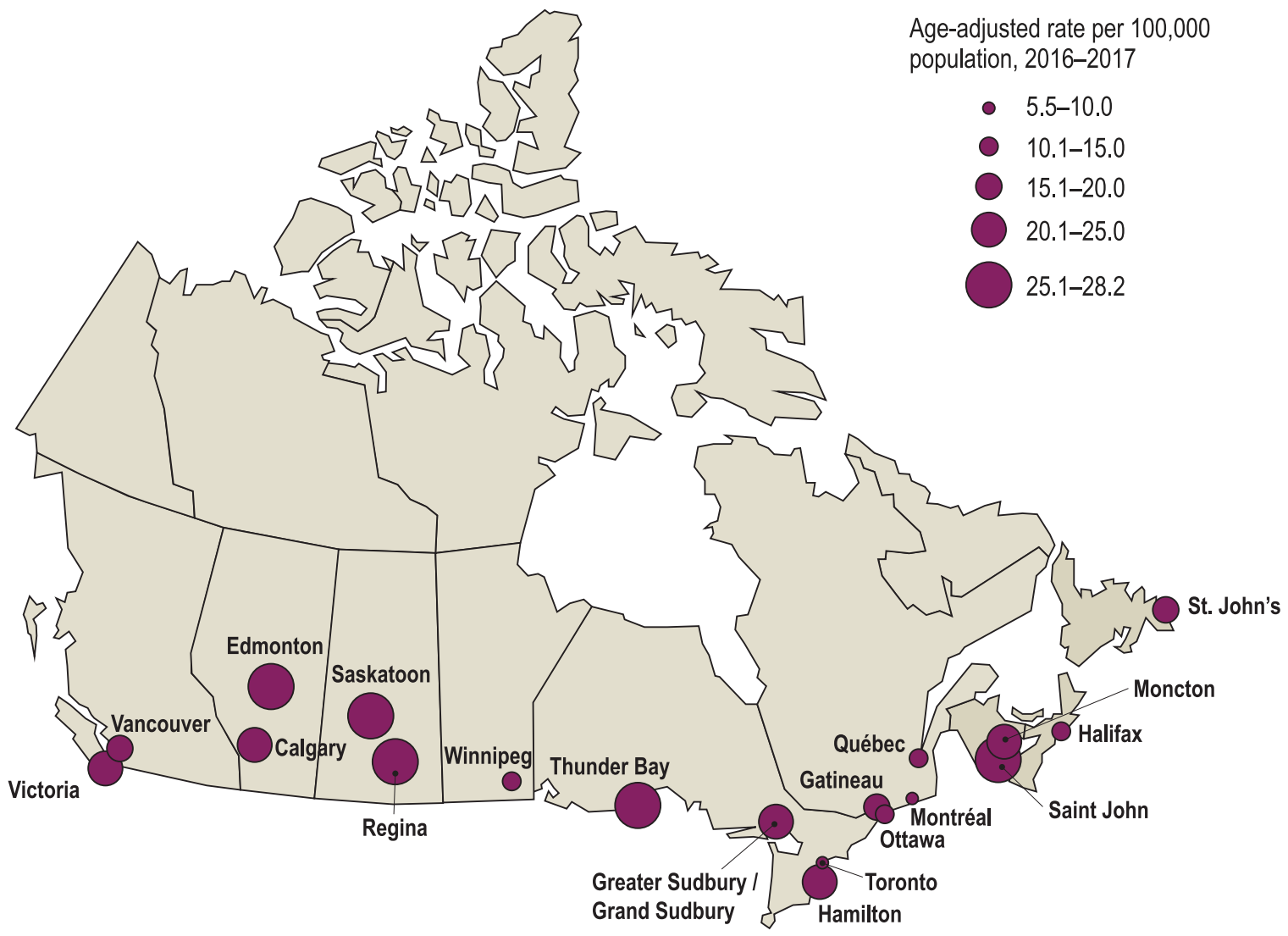

*Census metropolitan area is defined as an area consisting of one or more neighbouring municipalities situated around a core. A census metropolitan area must have a total population of at least 100,000 , of which 50,000 or more live in the core. Not all census metropolitan areas are shown in Figure 3.

Note: Quebec data are from 2015-2016, as this was the most recent data available at the time of the study.

Source: Hospital Morbidity Database, Canadian Institute for Health Information.

\section{Acknowledgements}

CIHI would like to acknowledge the input and advice of the Canadian Centre on Substance Use and Addiction and of the Public Health Agency of Canada. Please note that the analyses and conclusions in this publication do not necessarily reflect the views of the organizations mentioned above.

\section{References}

Canadian Institute for Health Information (CIHI). 2015. "Canadian Coding Standards for Version 2015 ICD-10-CA and CCI". Retrieved October 2, 2017.<https://www.cihi.ca/en/bul_icd10_feb15_pdf_en.pdf .

Government of Canada. 2017. "National Report: Apparent OpioidRelated Deaths in Canada (January 2016 to March 2017).” Retrieved October 2, 2017. <https://www.canada.ca/en/public-health/services/ publications/healthy-living/apparent-opioid-related-deaths-report2016-2017-december.html>.

\section{About the Authors}

This paper was prepared by the Prescription Drug Abuse (PDA) team at $\mathrm{CIHI}$. With support from Health Canada, the PDA team is developing a coordinated pan-Canadian approach to opioid monitoring, surveillance and reporting .

Vera Grywacheski, BA (Hons), MPH, is a senior analyst with the PDA team at $\mathrm{ClHI}$, Ottawa, Ontario. Vera can be reached by e-mail at vgrywacheski@cihi.ca

Shannon O'Connor, BA (Hons), MA, is a senior analyst with the PDA team at $\mathrm{ClHI}$, Ottawa, Ontario. Shannon can be reached by e-mail at soconnor@cihi.ca

Krista Louie, BSc, MSc, is a program lead with the PDA team at $\mathrm{ClHI}$, Ottawa, Ontario. Krista can be reached by e-mail at klouie@cihi.ca. 